\title{
Erratum to: Proteasome inhibition leads to early loss of synaptic proteins in neuronal culture
}

\author{
Natasha Bajic • Peter Jenner • \\ Clive G. Ballard $\cdot$ Paul T. Francis
}

Published online: 5 June 2013

(C) Springer-Verlag Wien 2013

\section{Erratum to: J Neural Transm (2012) 119:1467-1476 \\ DOI 10.1007/s00702-012-0816-9}

Unfortunately, crucial (contractually obligatory) text are missing in the acknowledgement section of the original article. The complete acknowledgments section should be:

This work was funded by the Edmond J. Safra Philanthropic Foundation. "This work was also funded by the
National Institute for Health Research (NIHR) Mental Health Biomedical Research Centre and Dementia Unit at South London and Maudsley NHS Foundation Trust and [Institute of Psychiatry] King's College London. This article presents independent research funded by the National Institute for Health Research (NIHR). The views expressed are those of the author(s) and not necessarily those of the NHS, the NIHR or the Department of Health."

The online version of the original article can be found under doi:10.1007/s00702-012-0816-9.

N. Bajic $\cdot$ C. G. Ballard $(\bowtie) \cdot$ P. T. Francis

Wolfson Centre for Age Related Diseases,

Guy's Campus, King's College London,

St. Thomas Street, London SE1 1UL, UK

e-mail: clive.ballard@kcl.ac.uk

\section{P. Jenner}

Institute of Pharmaceutical Science,

Hodgkin Building Guy's Campus,

King's College London, London SE1 1UL, UK 